\title{
A Survey on eLearning Content Standardization
}

\author{
Ricardo Queirós ${ }^{1}$ and José Paulo Leal ${ }^{2}$ \\ ${ }^{1}$ CRACS/INESC-Porto \& DI/ESEIG/IPP, Porto, Portugal \\ ricardo.queiros@eu.ipp.pt \\ ${ }^{2}$ CRACS/INESC-Porto \& DCC/FCUP, University of Porto, Portugal \\ zp@dcc.fc.up.pt
}

\begin{abstract}
Learning has been evolved in a gradual and consistent way. Along with this evolution several specialized and disparate systems appeared to fulfill the needs of teachers and students such as repositories of learning objects, intelligent tutors, or automatic evaluators. This heterogeneity poses issues that are necessary to address in order to promote interoperability among systems. Based on this fact, the standardization of content takes a leading role in the eLearning realm. This article presents a survey on current eLearning content standards. It gathers information on the most emergent standards and categorizes them according three distinct facets: metadata, content packaging and educational design.
\end{abstract}

Keywords: eLearning, standards, interoperability.

\section{Introduction}

In the last two decades we are witnessing to an impressive evolution of eLearning. Several types of eLearning systems appeared, from monolithic architectures to service oriented services aiming to cover all the needs of their users (e.g. staff, teachers, content authors, students) regarding academic, student or course management. One such system type is the Learning Management Systems (LMS) used to manage learning and track students' progress. This proliferation and heterogeneity of eLearning systems poses interoperability issues which are being considered by practitioners and educational institutions. In this context, these organizations have been creating standards and specifications to uniformize learning content and to develop interoperable tools and services [1].

The ultimate goal of this paper is to gather information on eLearning standards. For this study we selected several eLearning content standards and categorizes them based on three facets: metadata, content packaging and educational design. The first facet focuses on the description of learning resources. The second facet focuses on the organization and package of those resources for dissemination. The third facet deals with pedagogical issues in the presentation of those resources (e.g. design and sequence). 


\section{2 eLearning Standards}

The evolution of eLearning can be summarized by the transition of the early monolithic systems developed for specific learning domains to the new systems that can invoke specialized services and be plugged in any eLearning environments. These types of systems evolved from Content Management Systems (CMS). The CMS was introduced in the mid-1990s mostly by the online publishing industry. This type of system can be defined as a data repository that also includes tools for authoring, aggregating and sequencing content in order to simplify the creation, administration and access to online content [2]. The content is organized in small self-contained pieces of information to improve reusability at the content component level. These content components when used in the learning domain are called "learning objects" (LO) and the systems that manage them are called Learning Content Management Systems (LCMS) [3]. LO are context independent, transportable and reusable pieces of instruction that are digitally managed and delivered [4]. There are other definitions for Learning Objects (LO). Rehak \& Mason [4] define a learning object as: "a digitized entity which can be used, reused or referenced during technology supported learning".

In the eLearning context, standards are generally developed for the purposes of ensuring interoperability and reusability in systems and of the content and meta-data they manage. In this context, several organizations (IEEE, AICC, IMS, ADL) have been developed standards and specifications (Figure 1) [5] regarding the creation of standards, specifications, guidelines, best practices on the description and use of eLearning content.

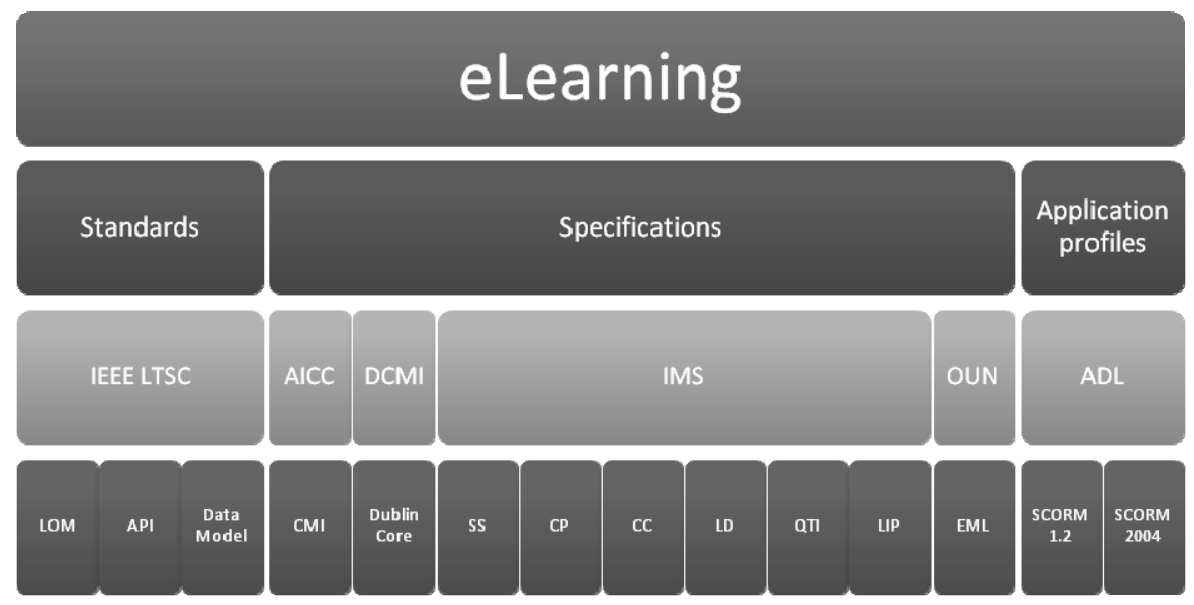

Fig. 1. eLearning Standards and Specifications [6]

In this study dozens of specifications were found. For the sake of readability we detail only the most prominent [7] organized in three facets: metadata, content package and educational design. 


\subsection{Metadata}

A learning object is composed by one or more educational resources. These resources are described by metadata. The most used metadata standard is the Learning Object Metadata (LOM). LOM is a double IEEE and IMS standard data model, usually encoded in XML, used to describe a learning object. LOM was a reaction to Dublin Core used by many repositories (e.g. DSpace). Dublin Core was judged to be too simple for adequately describing learning resources. The purpose of LOM is to support the reusability of learning objects, to aid discoverability, and to facilitate their interoperability, usually in the context of online learning management systems. LOM is integrated in a well known content packaging standard called IMS Content Packaging (IMS CP). The data model is organized in nine categories. The following table enumerates these categories based on a previous study [8].

Table 1. LOM data model categories

\begin{tabular}{|c|c|}
\hline Category & Description \\
\hline General & $\begin{array}{l}\text { Describe the learning object as a whole. This category includes } \\
\text { elements such as identifier, title, language, keywords. }\end{array}$ \\
\hline Lifecycle & $\begin{array}{l}\text { Describe features related to the history and current state of the LO } \\
\text { such as version, status, and contributors. }\end{array}$ \\
\hline Metametadata & $\begin{array}{l}\text { Group information about the metadata such as identifier, } \\
\text { contributors and language used in the metadata. }\end{array}$ \\
\hline Technical & $\begin{array}{l}\text { Describe the technical requirements and characteristics of the LO } \\
\text { such as MIME type, size, required software/hardware. }\end{array}$ \\
\hline Educational & $\begin{array}{l}\text { Describe educational and pedagogic characteristics of the LO such } \\
\text { as interactivity type, learning resource type, interactivity level, } \\
\text { semantic density, educational context, typical age range. }\end{array}$ \\
\hline Rights & $\begin{array}{l}\text { Describe the intellectual property rights and conditions of use for } \\
\text { the LO (whether or not any cost is involved, and whether } \\
\text { copyright and other restrictions apply). }\end{array}$ \\
\hline Relation & $\begin{array}{l}\text { Describe features that define the relationship between this LO and } \\
\text { others ('based on', 'part of', etc.). }\end{array}$ \\
\hline Annotation & $\begin{array}{l}\text { Provide comments on the use of the LO and information on when } \\
\text { and by whom the comments were created. }\end{array}$ \\
\hline Classification & $\begin{array}{l}\text { Describe where the LO can be classified within a particular } \\
\text { classification system. }\end{array}$ \\
\hline
\end{tabular}

These categories cover many facets of a LO. However, LOM was designed for general LO and does not to meet the requirements of specialized domains. Fortunately, it was designed to be straightforward to extend it. Next, we enumerate four ways that have been used [9] to extend the LOM model:

- combining the LOM elements with elements from other specifications;

- defining extensions to LOM elements while preserving its set of categories;

- simplifying LOM, reducing the number of LOM elements and its choices;

- extending and reducing simultaneously the number of LOM elements.

Based on the previous extension approaches, the IMS GLC created the Question \& Test Interoperability (QTI) specification. The IMS QTI specification describes a data 
model for the representation of questions (e.g. multiple choice, multiple response, fillin-the-blanks and short text questions) and tests data and their corresponding results reports. It extends the LOM with its own meta-data vocabulary as specified in the Meta-data and Usage Data document that describes a LOM profile suitable for use with assessment items and a separate data model for representing usage data (i.e., item statistics).

There are other metadata specifications, such as, the Dublin Core Metadata, which provides a simpler set of elements useful for sharing metadata across heterogeneous systems. At the present, the Dublin Education Working Group is extending the Dublin Core for the specific needs of the education community.

\subsection{Content Package}

Packaging is crucial to store eLearning material and reuse it in different systems. The most widely used content packaging format is the IMS Content Packaging (IMS CP). An IMS CP learning object assembles resources and meta-data into a distribution medium, typically an archive in ZIP format, with its content described in a manifest file in the root level. The manifest file - named imsmanifest.xml -adheres to the IMS $\mathrm{CP}$ schema and contains the following sections: Metadata - describes the package as a whole; Organizations - describes the organization of the content within a manifest; Resources - contains references to resources (files) needed for the manifest and metadata describing these resources; and Sub-manifests - defines sub packages.

The manifest uses the LOM standard to describe the learning resources included in the package. Recently, IMS Global Learning Consortium proposed the IMS Common Cartridge that adds support for several standards (e.g. IEEE LOM, IMS CP, IMS QTI, IMS Authorization Web Service) and its main goal is to shape the future regarding the organization and distribution of digital learning content. The latest revised version (1.1) was released in May 2011. The IMS CC manifest (Figure 2) includes references for two types of resources:

- Web Content Resources (WCR): static web resources that are supported on the Web such as HTML files, GIF/JPEG images, PDF documents, etc.

- Learning Application Objects (LAO): special resource types that require additional processing before they can be imported and represented within the target system. Physically, a LAO consists of a directory in the content package containing a descriptor file and optionally additional files used exclusively by that LAO. Examples of Learning Application Objects include QTI assessments, Discussion Forums, Web links, Basic LTI descriptors, etc.

There are other package specifications, mostly derived from the previous ones as application profiles. The term Application Profile generally refers to the adaptation, constraint, and/or augmentation of a metadata scheme to suit the needs of a particular community. A well know content packaging specification is SCORM that extends IMS CP with more sophisticated sequencing and Contents-to-LMS communication. These pedagogical contents and activities standards are detailed in the next subsection. 


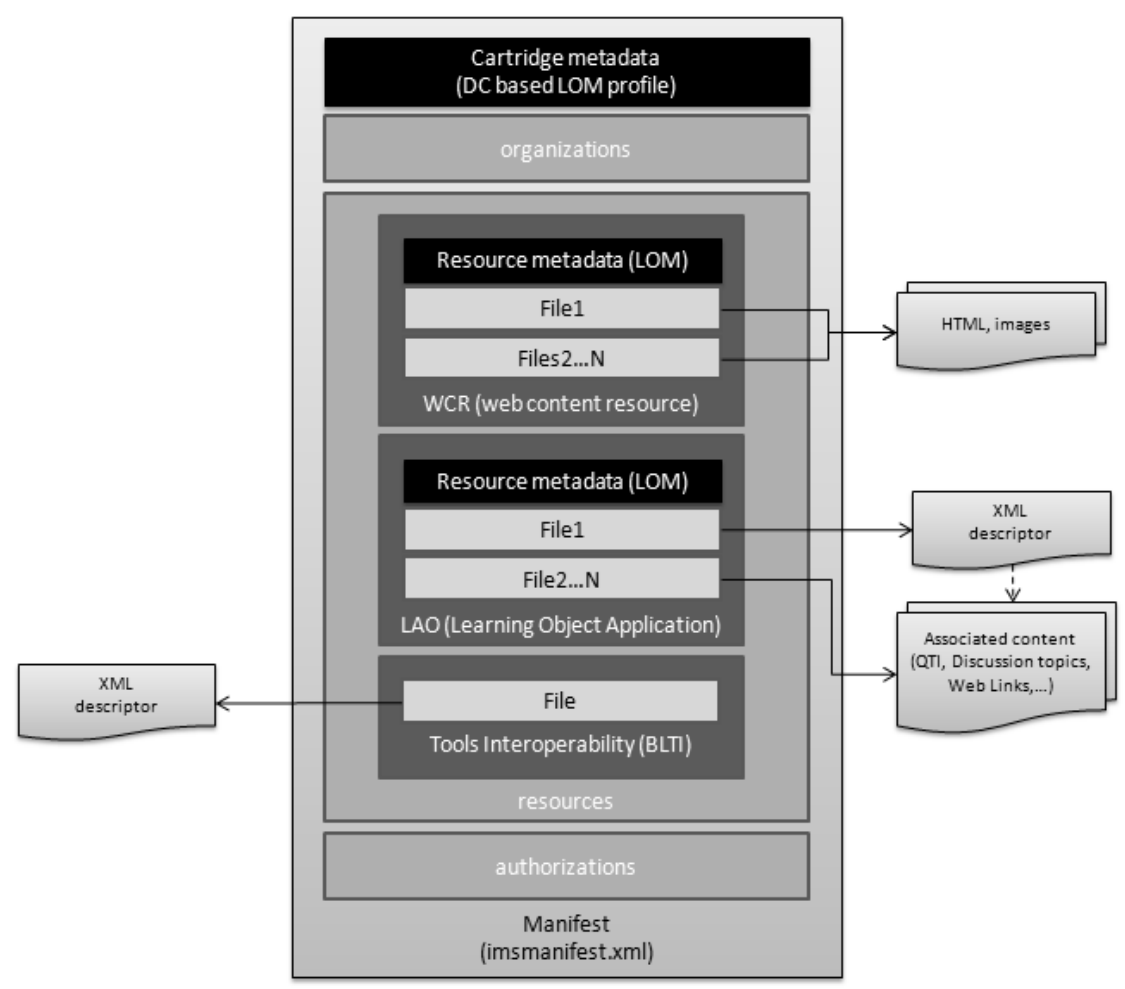

Fig. 2. IMS Common Cartridge package

\subsection{Educational Design}

Learning objects can be organized in items and an organization defines a path through those items. The IMS CP specification includes a manifest section called Organizations. This section can be used to design pedagogical activities and articulate the sequencing of instructions. By default, it uses a tree-based organization of learning items pointing to the resources (assets) included in the package. However, other standards could be accommodated in this section, such as IMS Simple Sequencing (IMS SS) and IMS Learning Design (IMS LD). These specifications aims to provide to the teachers mechanisms for coordination of the educational instructions based on students' profile making the instruction more dynamic and flexible.

The IMS LD specification is a meta-language for describing pedagogical models and educational goals. Several IMS LD-aware tools are available as players (e.g. CopperCore, .LRN) and authoring/export tools (e.g. Reload, LAMS).

The IMS SS is a specification used to describe paths through a collection of learning activities. The specification declares the order in which learning activities are to be presented to a learner and the conditions under which a resource is delivered during an eLearning instruction. Despite all these specifications, the design of more complex adaptive behaviour is still hard to achieve [10]. 


\section{Conclusions}

In this paper we present a study on eLearning content standards. We select the most prominent standards and specifications and categorize them in three facets. Based on this study we detect two issues that can hinder the proliferation of eLearning: fragmentation and complexity. The former is a typical issue in the technology realm. In this study we found dozens of specifications. While we presented only the most prominent it is important to state that standard fragmentation can reduce the amount of eLearning content available to any user, since educational players must support them. Other issue is related with the complexity of specifications. A good example is the IMS SS that few systems adhere. A modular approach (based on profiles) in the design of these specifications could help in the adequacy to real scenarios and domains and could facilitate the dissemination among communities.

\section{References}

1. Paepcke, A., Chang, C.K., Garcia-Molina, H., Winograd, T.: Interoperability for Digital Libraries Worldwide. Communications of the ACM 41(4), 33-43 (1998)

2. Nichani, M.: LCMS = LMS + CMS [RLOs] - How does this affect the learner? The instructional designer? (April 2009),

http: / /www.elearningpsot.com/articles/archives /

lcms_lms_cms_rlos

3. Leal, J.P., Queirós, R.: From eLearning Systems to Specialised Services. In: Chapter of EduJudge project book called "A New Learning Paradigm: Competition Supported by Technology". Sello Editorial (2010)

4. Rehak, D.R., Mason, R.: Keeping the learning in learning objects. In: Littlejohn, A. (ed.) Reusing Online Resources: a Sustainable Approach to e-Learning, pp. 22-30. Kogan Page, London (2003)

5. Friesen, N.: Interoperability and Learning Objects: An Overview of E-Learning Standardization. Interdisciplinary Journal of Knowledge and Learning Objects (2005)

6. Monroy, F.J.: Estándares en eLearning - Grupo CHICO, E.S. Informática, UCLM

7. Leal, J.P., Queirós, R.: A comparative study on LMS interoperability. In: Babo, R., Azevedo, A. (eds.) Higher Education Institutions and Learning Management Systems: Adoption and Standardization. IGI-Global (2011)

8. Duval, E.: Metadata Standards: What, Who \& Why. Journal of Universal Computer Science 7(7), 591-601 (2001)

9. Friesen, N.: Semantic and Syntactic Interoperability for Learning Object Metadata. In: Hillman, D. (ed.) Metadata in Practice, ALA Editions, Chicago (2004),

http: / /www. cancore.ca/

semantic_and_syntactic_interoperability.html (June 11, 2008)

10. Aroyo, L., Dolog, P., Houben, G., Kravcik, M., Naeve, A., Wild, F.: Interoperability in Personalized Adaptive Learning. Educational Tecnhnology \& Society (2006) 